$\Rightarrow$

\title{
Stress relief...
}

DOI:

10.1038/nrmicro1652

URLs

Neisseria meningitidis http://www.ncbi.nlm. nih.gov/entrez/query. $\mathrm{fcgi}$ ?db=genomeprj\&cmd= Retrieve\&dopt $=$ Overview \&list_uids $=12324$
Oxidative stress is a major cause of DNA damage and the mechanisms used by organisms to repair this damage are essential to their viability. Indeed, specialized immune cells take advantage of the destructive properties of oxidation to kill invading bacterial pathogens in what is a crucial component of the human immune system. A paper recently published in $E M B O$ Journal describes how one pathogen uses the coordinated action of two enzymes involved in DNA repair to counteract the oxidative damage inflicted by the host immune response.

Infections caused by the human pathogen Neisseria meningitidis are associated with significant morbidity and mortality worldwide. During disease progression, the pathogen is internalized by phagocytic cells and is subject to the reactive oxygen species produced by the cells. To minimize exposure to these oxidizing agents, the pathogen expresses superoxide dismutase, catalase and peroxidase. However, despite these protective mechanisms, DNA damage still occurs, so Carpenter et al. set out to investigate the contribution of base excision repair - the primary mechanism used by cells to counter the detrimental effects of oxidation - to meningococcal survival and pathogenesis. Initially, the authors identified two apurinic/apyrimidinic (AP) endonuclease paralogues in the genome of N. meningitidis. Rather than reflecting redundancy in important cellular activities, structural and biochemical characterization of these enzymes revealed that each have distinct and separate activities in DNA repair. One protein is a typical neisserial endonuclease (NApe) that excises abasic residues from the DNA backbone. The other protein is a specialized 3'-phosphodiesterase exonuclease (NExo) that has a different DNA repair function complementing the activity of NApe. To investigate the in vivo role of NApe and NExo in bacterial survival, strains were constructed that lacked each enzyme singly or both together. The authors demonstrated that the mutant strains were significantly more sensitive to oxidative stress when compared with the wild-type strain. Furthermore, an assessment of the ability of the mutant strains to cause bacteraemia - an essential step in meningococcal pathogenesis - revealed that both enzymes were required for bacterial survival during bloodstream infection.

These results clearly demonstrate the importance of NApe and NExo to oxidative stress resistance and meningococcal virulence. Interestingly, the authors also demonstrated that this combination of enzymes is present in a wide variety of bacterial species, a finding that points to the conserved importance of these coordinated DNA repair functions for prokaryotic survival under oxidative conditions.

David O'Connell

ORIGINAL RESEARCH PAPER Carpenter, E.P., et al. AP endonuclease paralogues with distinct activities in DNA repair and bacterial pathogenesis. EMBO J. 22 Feb 2007 (doi:10.1038/ sj.emboj.7601593) FURTHER READING

Davidsen, T. \& Tønjum, T. Meningococcal genome dynamics. Nature Rev. Microbiol. 4, 11-22 (2006)

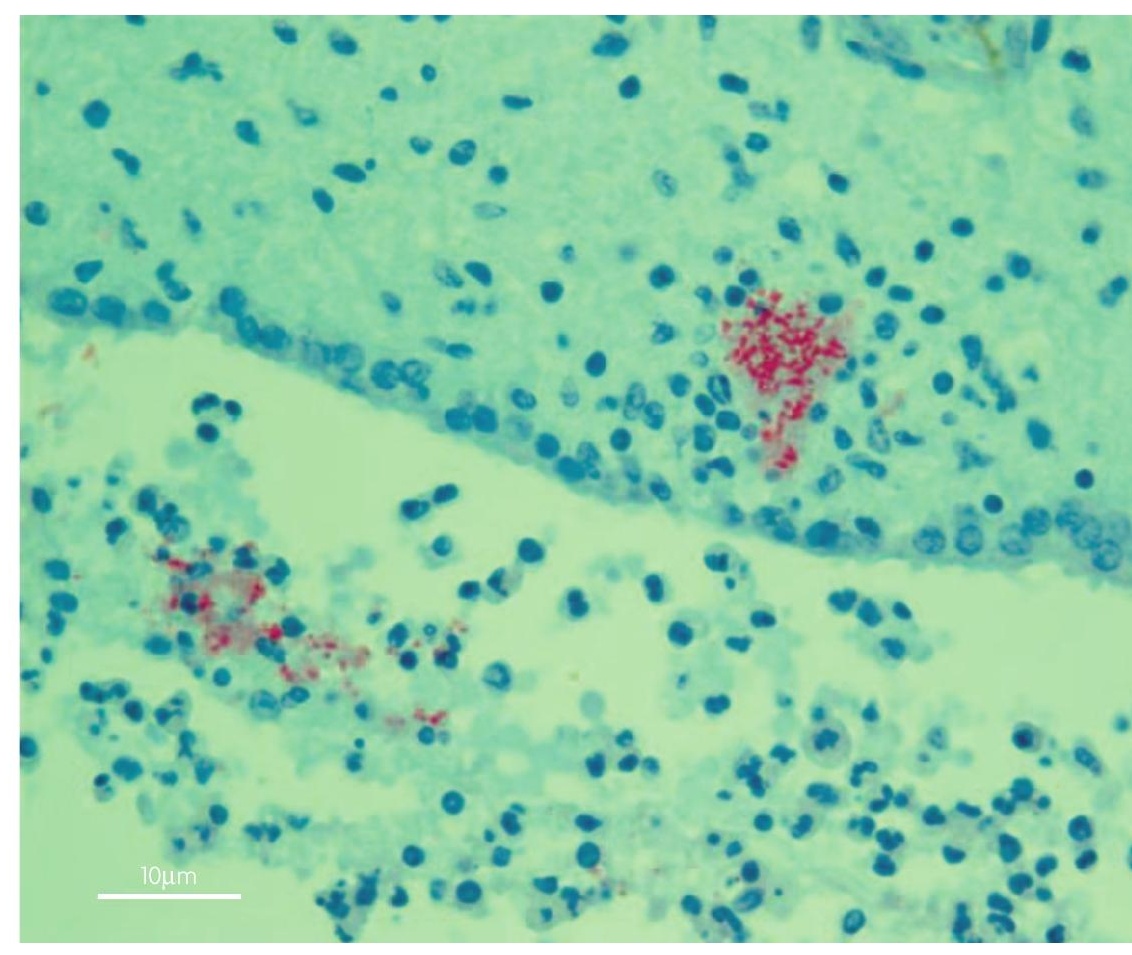

The image shows Neisseria meningitidis (stained red) in association with polymorphonuclear leukocytes (nuclei stained blue) in the cerebrospinal space of a patient with bacterial meningitis. 